\title{
A Raytracing Model for Wireless Propagation in Tunnels with Varying Cross Section
}

\author{
Camillo Gentile, Fabien Valoit, and Nader Moayeri \\ Advanced Network Technologies Division \\ National Institute of Standards and Technology \\ Gaithersburg, MD, USA \\ \{camillo.gentile, fabien.valoit, nader.moayeri\}@nist.gov
}

\begin{abstract}
Mandated by the 2006 United States Miner Act, reliable two-way communications in mines has drawn the interest of network engineers in recent years. Critical to the design of these systems is an accurate channel propagation model. Given the elementary geometry seen in most tunnels, models that approximate them as a rectangular waveguide have been developed. These models are extremely accurate in vehicular tunnels because - since the tunnel is typically cast from concrete - the cross section is uniform throughout and the surface roughness is negligible. Mines, however, do not conform to these two conditions. In this paper, we extend the waveguide model to tunnels with varying cross section and measurable surface roughness. The effectiveness of the proposed model is validated through in-house field measurements collected in a vehicular tunnel and in a coal mine. We show that while the original model performs well in the former, it falters in the latter. The extended model, however, predicts reliably in the mine as well.
\end{abstract}

Index Terms-Mine; surface roughness; convex; concave

\section{INTRODUCTION}

Prompted by two mining accidents which caused the deaths of 14 miners in West Virginia in January 2006, the United States Congress passed the most sweeping legislation in close to 30 years. Amongst other provisions, the 2006 Miner Act calls for two-way communications between underground miners and rescuers, as well as location tracking. Critical to the design of these systems is an accurate channel propagation model. In this context, recent years have seen the development of analytical models to predict the radio propagation of electromagnetic waves in tunnels [1], [2], [3]. The papers leverage the elementary geometry seen in most tunnels so as to approximate them as a rectangular waveguide, for which well-defined theory has been established. The theory permits closed-form expressions for the electromagnetic field by way of raytracing the reflected paths.

Associated with these analytical models are two main underlying assumptions. The first is that the radio waves behave like light, hence that the effects of diffraction can be ignored. This assumption is valid when the dimensions of the tunnel are much larger than the wavelength of the signal. This translates to a center frequency of a few hundred Megahertz for tunnels with cross-sectional dimensions on the order of several meters. The second assumption, of course, is that the tunnel is well approximated by a rectangular cuboid. For example, the results in [2], [3] are presented for a vehicular tunnel in France at center frequencies of $450 \mathrm{MHz}$ and $915 \mathrm{MHz}$. Since the cross section of the tunnel is close to rectangular - and moreover uniform throughout the shaft - the predictions are extremely accurate. However, when the cross section of the tunnel varies throughout the shaft $-\mathrm{a}$ prime example is in mines - the predictions break down. The breakdown does not arise simply from the variation in the dimensions, but also because they are typically much smaller than the dimensions of vehicular tunnels. As the dimensions decrease, the model is more sensitive to such variations as they represent a larger percentage of the nominal cross section.

To our knowledge, [3] is the most recently published work in this specific research area. Their model, intended for uniform cross sections, is described in Section II. In this paper, we propose two major improvements to it. The first is a raytracing model for tunnels with varying cross sections throughout the shaft. It is presented in Section III. The second improvement, in Section IV, is an extension of the model to include surface roughness. Again, as opposed to vehicular tunnels, this factor is much more significant in coal (iron, precious metals, etc.) mines due to the natural roughness of the rock. In order to substantiate the proposed model, we compare predicted results to in-house field measurements in two tunnels: a train tunnel and a coal mine. In Section V we show that in the mine - with varying cross section and surface roughness - our proposed model predicts reliably while the uniform model falters. The results are summarized in the Conclusions section.

\section{THE UNIFORM CROSS SECTION MODEL}

In this section, we present the raytracing model described in [3]. Figure 1(a) illustrates the model parameters. The cross-sectional profiles of the tunnel are given as $(a, b)$ in reference to the coordinate system with origin at the center of the tunnel, $(x=0, y=0, z=0)$, The cross section is said to be uniform because the profiles do not vary with $z$. The vertical $(v)$ and horizontal $(h)$ walls have electromagnetic properties $\left(\epsilon_{v}, \sigma_{v}\right)$ and $\left(\epsilon_{h}, \sigma_{h}\right)$ respectively, where $\epsilon$ denotes the permittivity and $\sigma$ the conductivity. The air in the tunnel has the permittivity of free space, $\epsilon_{0}$, and zero conductivity. The permeability of the air and the walls are all assumed to be equal to that of free space. The transmitter is positioned at $\left(x_{0}, y_{0}, 0\right)$ and the receiver at $(x, y, z)$, where $z$ is the range between the two. 


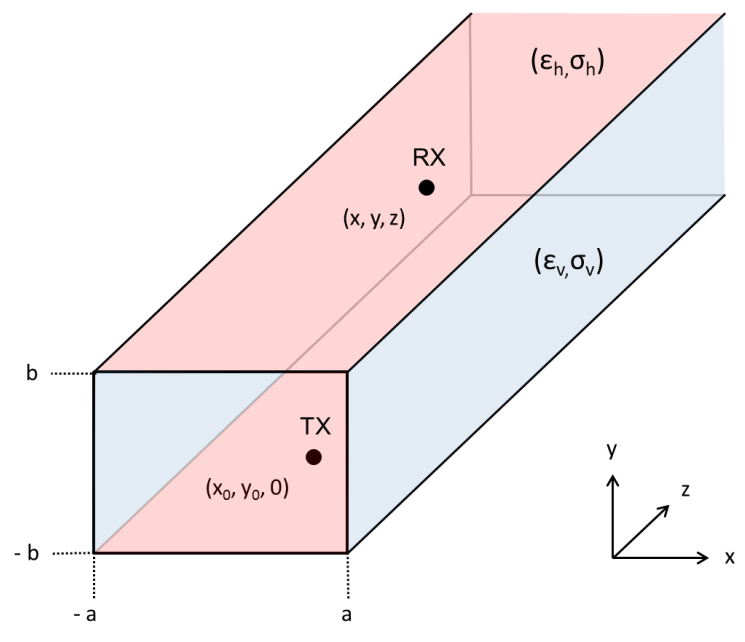

(a) The tunnel parameters

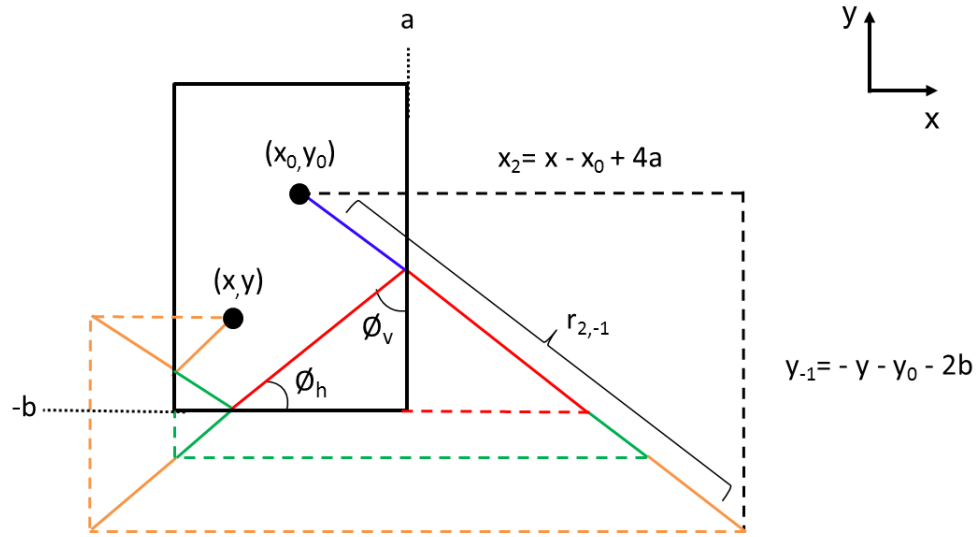

(b) The image reflection model

Fig. 1. The uniform cross section model. The vertical and horizontal walls have different electromagnetic properties and their profiles are symmetric about the respective axes. The cross section is uniform throughout the shaft of the tunnel.

The model traces all the rays (or paths) emitted from the TX which are received by the RX. Each path is indexed as $(p, q)$ according to the total number of $|p|$ reflections off the vertical walls and $|q|$ reflections off the horizontal walls. The reflections alternate between the positive and negative walls along the shaft. A positive value of $p$ indicates that the first reflection hits the positive vertical wall while a negative value indicates that the negative wall is hit first. The sign convention is the same for the horizontal walls.

The field attenuation associated with the collection of all propagation paths can be written as

$$
\alpha(x, y, z)=\sum_{p=-\infty}^{p=\infty} \sum_{q=-\infty}^{q=\infty}\left[\frac{\exp \left(-j k r_{p, q}\right)}{r_{p, q}}\right] \cdot R_{v}^{|p|} \cdot R_{h}^{|q|}
$$

where $r_{p, q}$ denotes the propagation distance of the indexed path. The bracketed term represents the complex attenuation due purely to distance traveled. As shown, it is inversely proportional to $r_{p, q}$. The numerator accounts for the phase shift, where $k=\frac{2 \pi f_{0}}{c}$ is the wavenumber, $f_{0}$ is the center frequency of the signal, and $c$ is the speed of light. The model assumes that reflection is the sole specular effect present in the tunnel. As expressed in (1), with each reflection off the walls the signal is attenuated by the corresponding reflection coefficient. The vertical and horizontal walls have reflection coefficients [4]:

$$
R_{v}=\frac{\cos \phi_{h}-\sqrt{\bar{k}_{h}-\sin ^{2} \phi_{h}}}{\cos \phi_{h}+\sqrt{\bar{k}_{h}-\sin ^{2} \phi_{h}}}
$$

and

$$
R_{h}=\frac{\bar{k}_{v} \cos \phi_{v}-\sqrt{\bar{k}_{v}-\sin ^{2} \phi_{v}}}{\bar{k}_{v} \cos \phi_{v}+\sqrt{\bar{k}_{v}-\sin ^{2} \phi_{v}}}
$$

where $\bar{k}_{v}=\left(\epsilon_{v}+\frac{\sigma_{v}}{j 2 \pi f_{0}}\right) / \epsilon_{0}$ and $\bar{k}_{h}=\left(\epsilon_{h}+\frac{\sigma_{h}}{j 2 \pi f_{0}}\right) / \epsilon_{0}$. The incident angles off the vertical and horizontal walls, $\phi_{v}$ and $\phi_{h}$ respectively, are calculated in the next subsection.
Implicit to the coefficients above is that the dipole antennas are vertically polarized. Analogous equations for horizontally polarized antennas are provided in [3]. In both cases, only the electromagnetic field in the main polarization direction is considered, assuming that the effects of cross polarization are minimal.

\section{A. The uniform image reflection model}

The propagation distance, $r_{p, q}$, is calculated through the image reflection model [5]. Fig. 1(b) shows the images for path $(p=2, q=-1)$ in the $(x, y)$-plane. For every vertical reflection, the image is displaced by a value $2 a$ in the $x$ direction; likewise, for every horizontal reflection, the image is displaced by a value $2 b$ in the $y$-direction. Then the total $x$-displacement for $p$ reflections is

$$
x_{p}=(-1)^{p} x-x_{0}+p \cdot 2 a
$$

and the total $y$-displacement for $q$ reflections is

$$
y_{q}=(-1)^{q} y-y_{0}+q \cdot 2 b .
$$

Knowing $z$ as well, the three can be combined as:

$$
r_{p, q}=\sqrt{x_{p}^{2}+y_{q}^{2}+z^{2}} .
$$

Also, from the $(x, y)$-displacements, the incident angles are calculated as

$$
\phi_{v}=\arcsin \frac{\left|x_{p}\right|}{r_{p, q}}
$$

and

$$
\phi_{h}=\arcsin \frac{\left|y_{p}\right|}{r_{p, q}} .
$$

Given the rectangular shape of the tunnel, the tunnel behaves as a rectangular waveguide. In fact it is shown in [3] how the raytracing model can be decomposed into a waveguide model expressed in terms of all the propagation modes. 


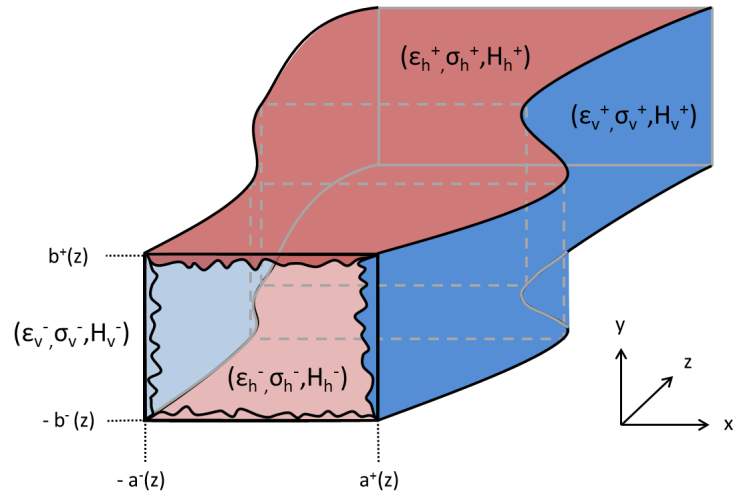

(a) The tunnel parameters

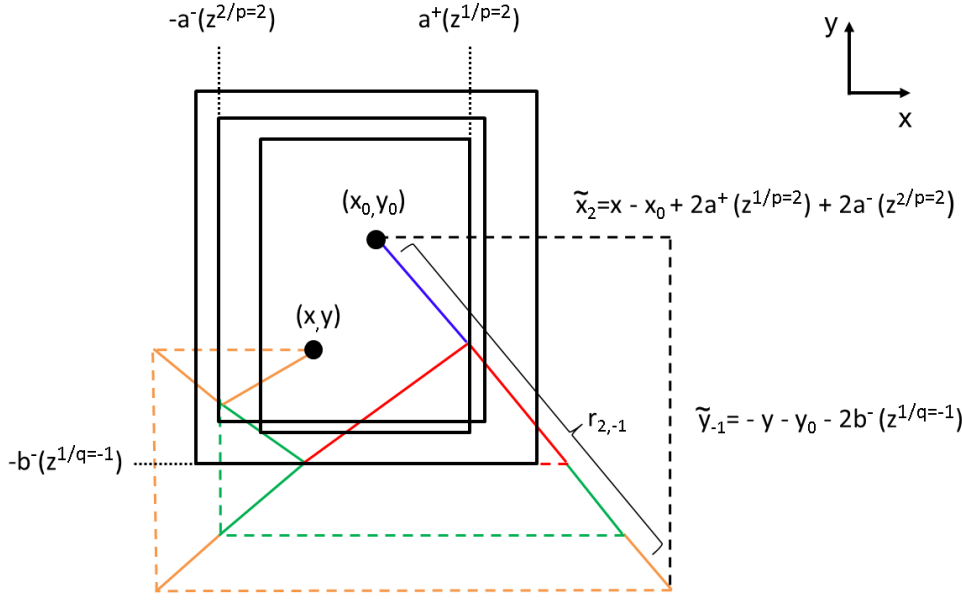

(b) The image reflection model

Fig. 2. The varying cross section model. The positive / negative walls may have different electromagnetic properties and their profiles may be asymmetric about the respective axes. The cross section varies throughout the shaft of the tunnel.

\section{THE VARYING CROSS SECTION MODEL}

In this section, we extend the uniform cross section model to account for large-scale variation in the tunnel profiles along the shaft. As a means to describe this variation, the vertical and horizontal profiles, $\left(a^{ \pm}(z), b^{ \pm}(z)\right)$, are written explicitly in terms of $z$. Figure 2(a) illustrates the model parameters. Notice that the positive / negative profiles are labeled separately, meaning that they may now be asymmetric, as shown. The attenuation is computed as in (1):

$$
\begin{aligned}
& \tilde{\alpha}(x, y, z)=\sum_{p=-\infty}^{p=\infty} \sum_{q=-\infty}^{q=\infty}\left[\frac{\exp \left(-j k r_{p, q}\right)}{r_{p, q}}\right] .
\end{aligned}
$$

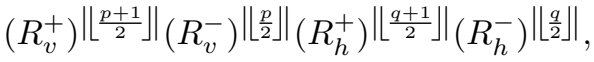

however the expressions for the displacements $x_{p}$ and $y_{p}$ in (4) and (5) must be modified as explained in the sequel. Also notice that positive / negative profiles may have different electromagnetic properties with designated reflection coefficients $\left(R_{v}^{ \pm}, R_{h}^{ \pm}\right)$given respectively from (2) and (3). Based on the values of $(p, q)$, (9) accounts for the number of times the signal is incident with each of the four walls.

\section{A. The varying image reflection model}

To see the effect of varying cross section on the displacements, we reconsider the image reflection model. Recall that for a uniform cross section, the $x$-displacement is augmented by $2 a$ with each reflection off the vertical wall. This translates to the total displacement in (4) for $p$ total reflections. Since the profile is uniform, where the reflections occur has no bearing on $x_{p}$. This is not the case when the profiles are varying. As such, we denote the vertical profile at the $\tilde{p}^{t h}$ out of $p$ total reflections as $a^{ \pm}\left(z^{\tilde{p} \mid p}\right)$, where $z^{\tilde{p} \mid p}$ is the $z$-coordinate at which the reflection occurs and the sign is given from the values of $\tilde{p}$ and $p$ as $\operatorname{sgn}(p)(-1)^{\tilde{p}+1}$. Now the $x$-displacement is augmented instead by $2 a^{ \pm}\left(z^{\tilde{p} \mid p}\right)$ with reflection $\tilde{p}$. Of course, the same relationship applies for the $y$-displacement with a total of $q$ reflections off the horizontal wall. Then the $(x, y)$-displacements follow as

$$
\tilde{x}_{p}=(-1)^{p} x-x_{0}+\operatorname{sgn}(p) \cdot 2 \sum_{\tilde{p}=\operatorname{sgn}(p)}^{p} a^{ \pm}\left(z^{\tilde{p} \mid p}\right)
$$

and

$$
\tilde{y}_{q}=(-1)^{q} y-y_{0}+\operatorname{sgn}(q) \cdot 2 \sum_{\tilde{q}=\operatorname{sgn}(q)}^{q} b^{ \pm}\left(z^{\tilde{q} \mid q}\right) .
$$

An example for $(p=2, q=-1)$ is illustrated in Fig. 2(b). As opposed to the uniform cross section in Fig. 1(b), shown here are the three cross sections corresponding to the three reflection points. Notice that the positive / negative profiles are asymmetric.

Compared to the uniform raytracing model, the varying model allows for wide-ranging tunnel profiles. However, in it, the cross section is modeled as rectangular - a stipulation upon which the image reflection model is based - such that the reflected angle is the same as the incident angle in the $(x, y)$-plane. In practice, most tunnels have either a circular, oval, or arched cross section; nevertheless the uniform model has been shown to give accurate results in those cases. The image reflection model also stipulates that the reflected angle be the same as in the incident angle in the $(x, z)$ - and $(y, z)$ planes, i.e. that the profiles are uniform - a condition which is obviously violated in the varying model. To minimize any violation, the orientation of the coordinate system should be chosen such that its axes lie as parallel as possible to the walls. So long as the local variation in the profiles is gradual - which is typical in most tunnels - the varying model can be applied. Results to substantiate this are presented in Section $\mathrm{V}$.

\section{B. Computing the reflection points}

We now turn our attention to a method for finding the reflection points. This method is illustrated by considering the simple example for $(p=1, q=0)$ shown in Figure 3 . 


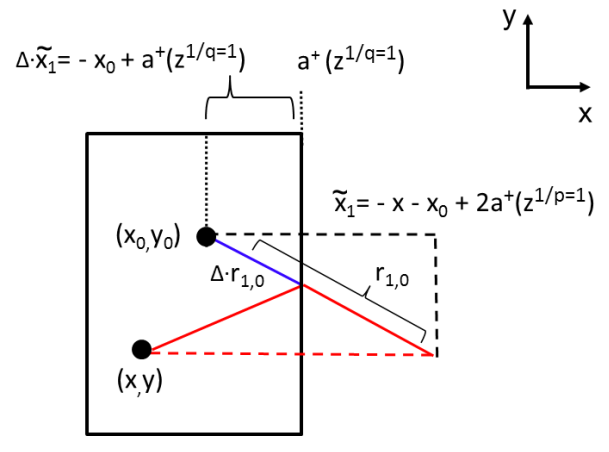

Fig. 3. The reflection point $z^{1 \mid q=1}$ for $(p=0, q=1)$. The marginal distance $\Delta \cdot r_{1,0}$ traveled from the transmitter to the reflection point can be expressed as a fraction $\Delta$ of the total distance $r_{1,0}$ traveled to the receiver.

The objective is to compute the value of $z^{1 \mid p=1}$ at which the single reflection occurs. This value then maps to $a^{+}\left(z^{1 \mid p=1}\right)$. The marginal distance traveled from the transmitter to the reflection point can be expressed as a fraction $\Delta$ of the total distance $r_{1,0}$, or

$$
\begin{aligned}
\Delta \cdot r_{1,0} & =\Delta \cdot \sqrt{\tilde{x}_{1}^{2}+\tilde{y}_{0}^{2}+z^{2}} \\
& =\sqrt{\left(\Delta \cdot \tilde{x}_{1}\right)^{2}+\left(\Delta \cdot \tilde{y}_{0}\right)^{2}+(\Delta \cdot z)^{2}}
\end{aligned}
$$

Of course the marginal distance traveled is along the same direction as the total distance traveled, i.e. along the direction of image $(p=1, q=0)$. Moving an amount $\Delta$ along this direction implies scaling each of the three vector components proportionally as in (12). In addition, it can be seen from the figure that the $x$-displacement of the image to the reflection point is $\Delta \cdot \tilde{x}_{1}=-x_{0}+a^{+}\left(z^{1 \mid p=1}\right)$. From it, the value of $\Delta$ can be recovered as

$$
\Delta=\frac{-x_{0}+a^{+}\left(z^{1 \mid p=1}\right)}{\tilde{x}_{1}} .
$$

By definition the $z$-coordinate of the image to the reflection point is given as $\Delta \cdot z=z^{1 \mid p=1}$. Finally, by substituting (13) into the latter, we arrive at

$$
z^{1 \mid p=1}=\left[\frac{-x_{0}+a^{+}\left(z^{1 \mid p=1}\right)}{\tilde{x}_{1}}\right] \cdot z .
$$

Through the same analysis, the $z$-coordinate can be indexed to any vertical or horizontal reflection point as

$z^{\tilde{p} \mid p}=\left[\frac{-x_{0}+\operatorname{sgn}(p) \cdot\left(a^{ \pm}\left(z^{\tilde{p} \mid p}\right)+2 \sum_{\varrho=\operatorname{sgn}(\tilde{p})}^{\tilde{p}-\operatorname{sgn}(\tilde{p})} a^{ \pm}\left(z^{\varrho \mid p}\right)\right)}{\tilde{x}_{p}}\right] \cdot z$,

$$
\tilde{p}=\operatorname{sgn}(p) \ldots p
$$

and

$$
z^{\tilde{q} \mid q}=\left[\frac{-y_{0}+\operatorname{sgn}(q) \cdot\left(b^{ \pm}\left(z^{\tilde{q} \mid q}\right)+2 \sum_{\varrho=\operatorname{sgn}(\tilde{q})}^{\tilde{q}-\operatorname{sgn}(\tilde{q})} b^{ \pm}\left(z^{\varrho \mid q}\right)\right)}{\tilde{y}_{q}}\right] \cdot z,
$$

Since the numerator in (15) involves all the vertical reflection points, the $|p|$ equations must be solved simultaneously; likewise, the $|q|$ equations for the horizontal reflection points in (16) must also be solved simultaneously.

\section{Convex versus concave profiles}

Because the set of equations in (15) is non-linear, it must be solved through numerical methods. The numerical methods for finding the $p$ reflection points involves searching along the vertical profiles. We first consider the case for which the profiles are convex. Figure 4(a) illustrates an example of convex positive / negative vertical profiles in the $(x, z)$-plane. The equations can be solved efficiently in the $|p|$-dimensional convex space through a gradient descent algorithm such as the Bisection method [6]. For uniform vertical profiles, the reflection points will occur at equal intervals along the $z$-axis; conversely, for varying vertical profiles, adjacent points occurring at consecutive positive and negative profile values below the respective profile averages will appear closer together - since the ray travels a shorter distance between them - while those occuring at values above the average will appear farther apart. At initialization, the points are ordered with equal spacing between each other; the gradient descent algorithm effectively scales the spacing according to the profile values at which they occur. While the range of any point is limited to $0<z^{\tilde{p} \mid p}<z$, additional constraints limit the search space. Specifically, e.g. for $p$ positive, knowing that the $\tilde{p}^{t h}$ reflection will occur before the $(\tilde{p}+1)^{t h}$ reflection along the shaft implies $z^{\tilde{p} \mid p}<z^{\tilde{p}+1 \mid p}$, $\tilde{p}=1 \ldots p-1$.

Fig. 4(b) illustrates an example of concave positive / negative vertical profiles in the $(x, z)$-plane. In tunnels with concave profiles, solving for the set of equations in turn entails a search in a concave space, which in general is much less efficient than in its counterpart. However, since the search is just between adjacent points - so long as the associated profiles are convex in between - finding the global optimum requires no additional complexity. This is often the case because, as mentioned earlier, in most tunnels the variation in the the wall profiles is gradual. Notwithstanding, for $|p|=1$ the point could fall at any point in the range, indeed requiring a concave search. While in a single dimension a concave search is still quite efficient, it becomes more and more complex as the number of reflection points increases; however, the increased point density tightens the spacing between adjacent points, limiting the search space further and compensating to some degree for the greater complexity.

There is actually a more important issue in concave tunnels (15) than the efficiency of the numerical method. In convex 


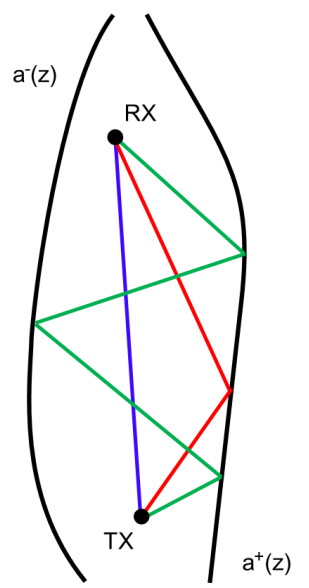

(a) Convex

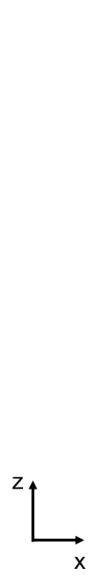

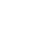

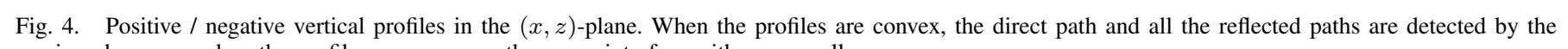
receiver, however, when the profiles are concave, they may interfere with some walls.

tunnels the transmitter and receiver are always in line-ofsight of each other. It follows that the direct path and all the reflected paths will pass unobstructed by the tunnel walls, as shown in Fig. 4(a). In concave tunnels, however, this is not always the case. When the direct path is obstructed because the typical materials and the thickness of the tunnel walls (e.g. concrete, coal) permit virtually no penetration it will not be detected by the receiver. It is easy to determine through simple geometry whether this is the case; if so, the associated term $(p=0, q=0)$ should be eliminated from the sum in (9). In general, once path $(p, q)$ is computed, it should be checked through the same geometry and eliminated if obstructed. Note that as the reflection order increases, so does the incident angle, allowing the signal to more readily reflect around concave segments, as shown in Fig. 4(b). The paths for $q=0$ (blue) and $q=-1$ (red) are obstructed, however not for $q=-3$ (green). For gradual profiles, unobstructed paths with lower reflections imply that the higher-order paths are also unobstructed and hence need not be checked.

\section{Surface Roughness}

Surface roughness, which may be viewed as small-scale or local variation in the dimensions of the cross section, can account for significant attenuation of the signal upon incidence with a wall, especially at higher frequencies. At higher frequencies, it can be seen from (9) that the phase of an individual path varies more as a function of path length. As the paths scattered from different parts of the rough surface combine with correspondingly different lengths, the phases add destructively to create interference, attenuating the signal [7].

The conventional metric for surface roughness is the quantity $H$, which is the root mean square of the deviation in height of the profile from its large-scale value. Dependent upon the incident angle, the effective height is reduced to $H \sin \phi$. The surface roughness coefficients of the signal upon incidence can be expressed as [7]

$$
S_{v}^{ \pm}=\exp \left(-2\left(k H_{v}^{ \pm} \sin \phi_{v}\right)^{2}\right)
$$

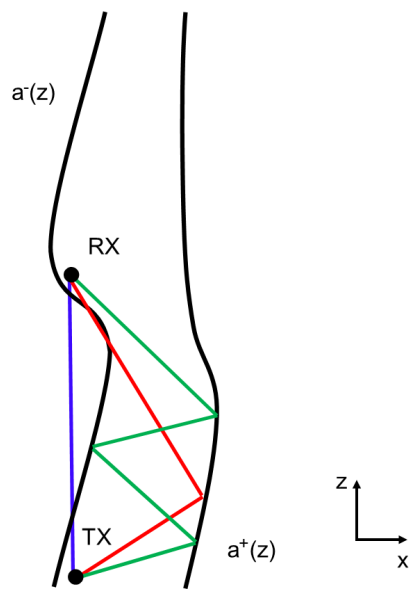

(b) Concave
TABLE I

TUNNEL PARAMETERS

\begin{tabular}{|l||c|c|}
\hline & $\begin{array}{c}\text { Train } \\
\text { tunnel }\end{array}$ & $\begin{array}{c}\text { Coal } \\
\text { mine }\end{array}$ \\
\hline \hline $\mathrm{a}(\mathrm{m})$ & 2.44 & $1.22-1.52$ \\
\hline $\mathrm{b}(\mathrm{m})$ & 3.12 & $0.84-1.14$ \\
\hline$\epsilon\left(\frac{F}{m}\right)$ & $7 \cdot \epsilon_{0}$ & $4 \cdot \epsilon_{0}$ \\
\hline$\sigma\left(\frac{S}{m}\right)$ & 0.0150 & 0.0007 \\
\hline $\mathrm{H}(\mathrm{cm})$ & 0 & 20 \\
\hline
\end{tabular}

and

$$
S_{h}^{ \pm}=\exp \left(-2\left(k H_{h}^{ \pm} \sin \phi_{h}\right)^{2}\right)
$$

where $\left(H_{v}^{ \pm}, H_{h}^{ \pm}\right)$are the respective roughness metrics for the positive / negative vertical and horizontal walls. These metrics are depicted in Figure 2(a). Notice, as explained earlier, that the attenuation coefficients increase with center frequency. However, in the seminal paper [8] on wireless propagation in tunnels, Emslie et al. indicate exactly the opposite. This is because in their development they consider only the fundamental propagation modes in the waveguide model while here they are all considered.

By modeling the effect of surface roughness, we arrive at a new expression for the attenuation at the receiver:

$$
\begin{array}{r}
\hat{\alpha}(x, y, z)=\sum_{p=-\infty}^{p=\infty} \sum_{q=-\infty}^{q=\infty}\left[\frac{\exp \left(-j k r_{p, q}\right)}{r_{p, q}}\right] . \\
\left.\left(R_{v}^{+} S_{v}^{+}\right)^{\left\lfloor\frac{p+1}{2}\right\rfloor}\left(R_{v}^{-} S_{v}^{-}\right)^{\left\lfloor\left[\frac{p}{2}\right\rfloor\right.}\left(R_{h}^{+} S_{h}^{+}\right)^{\left\lfloor\frac{q+1}{2}\right\rfloor}\right|_{\left(R_{h}^{-} S_{h}^{-}\right)^{\left\lfloor\left\lfloor\frac{q}{2}\right\rfloor\right.} .}
\end{array}
$$

\section{EXPERIMENTAL RESULTS}

In this section, we compare the uniform and varying models against field measurements collected in two different types of tunnel. In our experiments, the transmitter and receiver antennas were mounted on tripods and both positioned at the midpoint of the tunnel cross section. The receiver was initially placed at $z=1 \mathrm{~m}$. Then the range from the transmitter was increased progressively while maintaining the same 


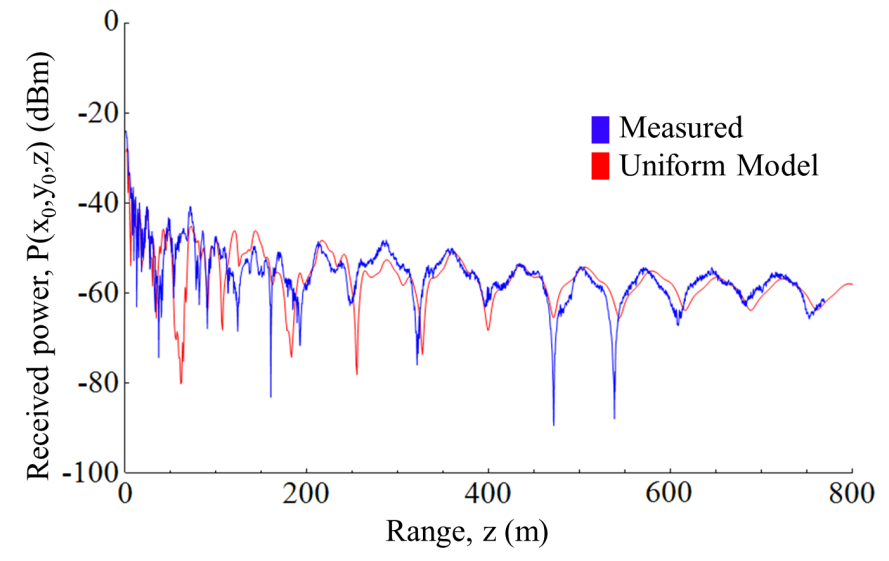

(a) Train tunnel

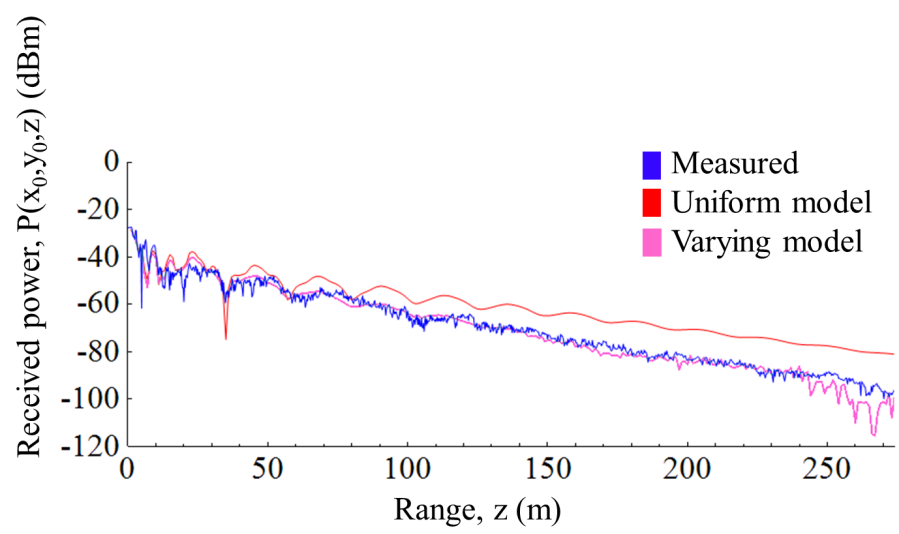

(b) Coal mine

Fig. 5. Experimental results at center frequency $f_{0}=915 \mathrm{MHz}$. In the train tunnel with uniform cross section, the uniform model reliably tracks the measured received power; however, in the coal mine with varying cross section, it falters while the varying model performs well.

position in the $(x, y)$-plane, hence for $x=x_{0}$ and $y=y_{0}$. At each point, the received power was measured across the frequency band of interest using a spectrum analyzer. Both the transmitter and the receiver were equipped with vertically polarized omni-directional antennas and the transmit power was set to $P^{T X}=1.5 \mathrm{dBm}$. Knowing the transmit power, the received power predicted from the attenuation model is given through Friis formula [4] as

$$
P(x, y, z)=P^{T X} \cdot\left(\frac{c}{4 \pi f_{0}}\right)^{2}|\alpha(x, y, z)|^{2} .
$$

The measured received power and predicted received power, $P\left(x_{0}, y_{0}, z\right)$, were then compared.

The first tunnel was the Sorrento Tunnel in Plummer, Idaho. The train tunnel is constructed from concrete, thus cast with negligible surface roughness; most importantly, the structure has a uniform profile throughout. Since the ceiling is arched, the vertical profile parameter, $b$, was computed such that its value corresponds to the rectangular cross section which best approximates the actual one in the least-squares sense. Also, the material properties were the same for all four walls. Table I shows the parameters for both tunnels. The second tunnel was an experimental coal mine in Pittsburgh, Pennsylvania. There is a number of significant differences between the two tunnels. First of all the dimensions of the mine range from a fourth to half the size of the train tunnel. Secondly, the profile parameters vary up to $35 \%$ through the mine shaft while in the train tunnel they are constant. Finally, the surface roughness of the coal is $20 \mathrm{~cm}$.

Fig. 5(a) displays the measured versus the predicted received power as a function of $z$ in the train tunnel. Observe that, despite the rectangular approximation, the uniform model predicts very reliably - both in the near field of the transmitter as well as in the far field - tracking the peaks and valleys where the paths respectively combine constructively and destructively throughout the shaft. In view of the varying profiles coupled with the surface roughness of the coal mine,
Fig. 5(b) shows that the uniform model falters. However, the varying model still predicts reliably.

\section{CONCLUSIONS}

In this paper, we have proposed a novel analytical model for the propagation of electromagnetic waves in tunnels with varying cross section. Tunnels with both convex and concave profiles have been considered. The model has application, in particular, to the design of wireless communications in mines. The major underlying assumption is that the variance in the cross section rolls out gradually along the shaft. The proposed model has been validated with in-house field measurements and has been shown to predict reliably in a coal mine. Further work includes refining the model for more complex tunnel structures, including cross cuts.

\section{REFERENCES}

[1] Mahmoud, S.F., "Modal Propagation of High Frequency Electromagnetic Waves in Straight and Curved Tunnels within the Earth," Journal of Electromagnetic Waves and Applications, vol. 19, no. 12, pp. 16111627, 2005.

[2] Dudley, D.G.; Lineard, M.; Mahmoud, S.F.; Degauque, P., "Wireless Propagation in Tunnels," IEEE Antennas and Propagation Magazine, vol. 49, no. 2, April 2007.

[3] Sun, Z.; Akyildiz, I.F., "Channel Modeling and Analysis for Wireless Networks in Underground Mines and Road Tunnels, IEEE Transactions on Communications, vol. 58, no. 6, pp. 1758-1768, June 2010.

[4] Frankl, D.R., Electromagnetic Theory, Prentice-Hall, Englewood Cliffs, NJ, 1986

[5] Valenzuela, R.A., "A ray tracing approach to predicting indoor wireless transmission," IEEE Vehicular Technology Conf., pp. 214-218, May 1993.

[6] Burton, R.L.; Fairies, D.J., Numerical Analysis, Seventh Edition, Brooks/Cole, Pacific Grove, CA, 2001.

[7] Beckmann, P. and Spizzichino, A., The Scattering of Electromagnetic Waves from Rough Surfaces, Macmillan, New York, 1963.

[8] Emslie, A.G.; Lagace, R.L.; Strong, P.F., "Theory of the Propagation of UHF Radio Waves in Coal Mine Tunnels," IEEE Trans. on Antennas and Propagation, vol. 23, no. 2, March 1975. 